Editorial

\title{
Lipid profile in children with Nephrotic syndrome
}

\author{
Chavan S. ${ }^{1}$, Salunkhe S. ${ }^{2}$, Singh A. ${ }^{3}$, Agarkhedkar S. ${ }^{4}$, Suhas Sodal ${ }^{5}$, Jadhav R. ${ }^{6}$ \\ ${ }^{1}$ Dr. Sanjay Chavan, Professor, ${ }^{2}$ Dr. Shradha Salunkhe, Associate Professor, ${ }^{3}$ Dr. Anshuman Singh, Senior Resident, \\ ${ }^{4}$ Dr. Sharad Agarkhedkar, Professor and Head of Department, ${ }^{5}$ Dr. Suhas Sodal, Assistant Professor, ${ }^{6}$ Dr. Renuka Jadhav, \\ Professor, all authors are affiliated with Dr. D.Y. Patil Medical College, Hospital And Research Center, Dr. D.Y.Patil \\ Vidyapeeth Society, Pimpri, Pune. Maharashtra, India.
}

Corresponding Author: Dr. Shradha Salunkhe, Associate Professor, Dr D.Y. Patil Medical College Hospital and Research Center, Pimpri, Pune, Maharashtra. India. Email Id: salunkheshradha@gmail.com

\begin{abstract}
Background: To study the derangement of serum lipid profile in children 2 to 12 years with nephrotic syndrome. Methods: 50 children 2 to 12 years with nephrotic syndrome were identified. Patients were classified as remission, relapse and newly diagnosed. Lipid Profile was measured. Patients were followed-up after 4 weeks of steroid therapy. Results: Out of all the 50 subjects screened, 25 (50\%) subjects had high total cholesterol, 26 (52\%) had high triglyceride, $8(16 \%)$ had abnormal HDL cholesterol and $25(50 \%)$ had high LDL cholesterol. After 4 weeks of steroid therapy though there was significant reduction in lipid components. Conclusion: Our study shows that in nephrotic syndrome, there is generalised hyperlipidemia and hypoalbuminemia. Serum cholesterol, triglycerides and LDL cholesterol were deranged in almost all subjects. In cases in relapse after 4 weeks of steroid therapy there is persistent raised total cholesterol, triglycerides and LDL cholesterol, which may predispose to the development of atherosclerosis in near future.
\end{abstract}

Keywords: Nephrotic syndrome, Hyperlipidemia, hypoalbuminemia, steroid.

\section{Introduction}

Nephrotic syndrome is primarily a paediatric disorder and is 15 times more common in children than adults. The incidence is $2-3 / 100,000$ children per year; and the majority of affected children will have steroid-sensitive minimal change disease. The characteristic features of nephrotic syndrome are heavy proteinuria $(>3.5 \mathrm{~g} / 24 \mathrm{hr}$ in adults or $40 \mathrm{mg} / \mathrm{m}^{2} / \mathrm{hr}$ in children), hypoalbuminemia $(<2.5 \mathrm{~g} / \mathrm{dl}$ ), oedema, and hyperlipidemia (serum cholesterol $>200 \mathrm{mg} / \mathrm{dl}$ ). Hyperlipidemia is recognised as a common finding in patients with nephrotic syndrome since 1917, when hyper cholesterolemia was described as a feature of nephrotic syndrome [1]

Hyperlipidemia is usually observed during the active phase of the disease and disappears with resolution of proteinuria. However, it may persist in some cases, leading to increase risk of atherosclerosis in later life and development of progressive renal injury [2]. Hence close monitoring of lipid levels during remission of nephrotic syndrome is necessary to select high risk patients [3] Children with Nephrotic Syndrome are at an increase risk for cardio vascular disease due to the

Manuscript received: $6^{\text {th }}$ June 2018

Reviewed: $16^{\text {th }}$ June 2018

Author Corrected: $24^{\text {th }}$ June 2018

Accepted for Publication: $30^{\text {th }}$ June 2018 hyperlipidemia present in them. Hyperlipidemia is not always connected with nephrotic disease activity and may sometimes persist for long time, especially in frequently relapsing nephrotic syndrome. Though after steroid treatment the lipid profile shows lower values than in active state, no normalisation of indices were observed in remission [4].

The present study is designed to study the spectrum of serum lipid profile abnormalities in children with nephrotic syndrome.

\section{Materials and Methods}

Aims and Objectives: To study the derangement of serum lipid profile in children 2 to 12 years with nephrotic syndrome.

Type of study: Prospective case study

Sample size: 50 cases fulfilling the inclusion criteria

Place of study: Dr. D.Y.Patil Medical College, Hospital and Research Center, Pimpri, Pune- 18

Inclusion Criteria: Following parameters were considered for inclusion of cases in present study: 
Editorial

- Age group between 2 years to 12 years

- Idiopathic nephrotic syndrome

- Relapsing nephrotic syndrome

- Frequent relapses nephrotic syndrome

- Steroid dependence nephrotic syndrome

- Steroid resistant nephrotic syndrome

\section{Exclusion Criteria}

- Age less than 2 years and more than 12 years

- Familial hyperlipidemia

- Presence of any disease other than Nephrotic syndrome like persistent hypertension, renal tumours, cardiovascular disorders etc

Methodology: All the children fulfilling the inclusion criteria were identified and selected for study after obtaining written informed consent. The sample population were recruited from the outpatient clinic or in patient's ward of Dr. D. Y. Patil Medical College, Hospital and Research Centre, Pimpri, Pune. A detailed history was taken and samples were taken for further investigations. Patients were classified as remission, relapse and newly diagnosed.

Children with oedema, low serum albumin and urinary protein of more than $40 \mathrm{mg} / \mathrm{m} /$ hour or $3+/ 4+$ protein were considered as nephrotic syndrome or relapse.

Patients were considered in remission when urine albumin was nil or trace or proteinuria less than 4 $\mathrm{mg} / \mathrm{m} 2$ /hour for three consecutive days. Patients were classified as remission, relapse and newly diagnosed.

Blood was collected in fasting state in the early morning and the samples were analysed for serum total proteins, serum albumin, serum globulin, blood urea, serum creatinine and lipid profile (total cholesterol, triglycerides, LDL, VLDL, HDL). Lipid profile was measured at the admission to the hospital and again in remission.
The Serum Lipid was measured by: Total cholesterol: measured by CHOD-PAP Method, Triglycerides: measured by GPO-TINDER Method, HDL and LDL Cholesterol: measured by Polyvinyl Sulfonic Acid (PVS) and Polyethylene Glycol Methyl Ether (PEGME) coupled classic precipitation method.

Serum proteins were measured by Biurate Method. The renal functions were measured by Urease Method. Creatinine clearance was calculated by: CockcroftGault equation.

Creatinine clearance $=[140 \text {-age }(\text { years })]^{*}$ weight $\left.(\mathrm{kg})\right] /$ $[72 *$ s.creatinine $(\mathrm{mg} / \mathrm{dl})]$

Multiply by 1 for male

0.85 or female

The UPCR: Urine protein was measured by - Pyragalol method and Urine creatinine was measured by Jaffe w/o deproteinization. (UPCR- urine protein creatinine ratio)

Patients in relapse and newly diagnosed cases were followed-up after 4 weeks of steroid therapy and samples for serum protein and serum lipid were taken.

Treatment protocol: First episode: $60 \mathrm{mg} / \mathrm{m} 2$ /day daily (maximum dose $80 \mathrm{mg}$ divided into 2-3 doses) prednisolone for 6 weeks, followed by $40 \mathrm{mg} / \mathrm{m} 2$ /day alternate day as a single morning dose for 6 weeks.

Relapse cases $60 \mathrm{mg} / \mathrm{m} 2$ /day daily (maximum dose 80 $\mathrm{mg}$ divided into 2-3 doses) prednisolone until child enters remission, followed by $40 \mathrm{mg} / \mathrm{m} 2$ /day alternate day as a single morning dose for 6 weeks.

Stastical analysis: The collected data were analysed using the SSPS software (Statistical Package for the Social Sciences, version 2.1). $p$ values less than 0.05 were considered statistically significant.

\section{Results}

In the study there were $40 \%$ of children in the $2-4$ years of age group, $36 \%$ between $4-8$ years of age group and $24 \%$ between $8-12$ years. In the study there were $56 \%$ males and $44 \%$ females, suggesting a male predominance.

Cases were classified into relapse, remission and new cases. $46 \%$ cases were in relapse, $40 \%$ in remission and $14 \%$ were newly diagnosed cases. Out of 23 subjects in relapse 18 (78.25\%) were frequent relapsers, $4(17.49 \%)$ of infrequent relapse while $1(4.35 \%)$ was having steroid resistance.

Out of 50 cases screened 29 (58\%) subjects had periorbital oedema, 21 (42\%) had ascites, 14 (28\%) had oliguria, 13 $(26 \%)$ had generalised oedema, $9(18 \%)$ had pallor, $4(8 \%)$ had hypertension and $1(2 \%)$ had peritonitis. 
Editorial

Table-1: Derangement of serum lipids of children in study group.

\begin{tabular}{|c|c|c|}
\hline Lipid profile & No of cases & Percentage $(\mathbf{n}=\mathbf{5 0})$ \\
\hline Sr. Cholesterol & 25 & 50 \\
\hline Sr. Triglyceride & 26 & 52 \\
\hline Sr. HDL & 8 & 16 \\
\hline Sr. LDL & 25 & 50 \\
\hline
\end{tabular}

Out of all the 50 subjects screened $25(50 \%)$ had elevated total cholesterol levels, $26(52 \%)$ had elevated triglyceride levels, $8(16 \%)$ had deranged HDL cholesterol levels and $25(50 \%)$ had elevated LDL Cholesterol levels.

Table-2: Correlation between Sr. Albumin and lipid profile in study group.

\begin{tabular}{|c|c|c|}
\hline Correlation between Sr. Albumin & r Value & P Value \\
\hline Sr. Cholesterol & -0.74 & $<0.0001$ \\
\hline Sr. Triglyceride & -0.43 & $<0.005$ \\
\hline Sr. HDL & -0.31 & $<0.05$ \\
\hline Sr. LDL & -0.49 & $<0.0001$ \\
\hline
\end{tabular}

There is inverse correlation of total cholesterol, triglyceride, HDL and LDL cholesterol with serum albumin and is statistically significant.

Table-3: Correlation between Creatinine clearance and lipid profile in study group.

\begin{tabular}{|c|c|c|}
\hline Correlation between Creatinine clearance & r Value & P Value \\
\hline Sr. Cholesterol & 0.12 & $>0.05$ \\
\hline Sr. Triglyceride & 0.24 & $>0.05$ \\
\hline Sr. HDL & -0.04 & $>0.05$ \\
\hline Sr. LDL & 0.19 & $>0.05$ \\
\hline
\end{tabular}

There was positive correlation of total cholesterol, triglyceride and LDL cholesterol with creatinine clearance but was statistically not significant. There was negative correlation of HDL cholesterol and was statistically not significant.

Table-4: Showing number of subjects with derangement of lipid parameters Pre steroid after 4 weeks of steroid therapy.

\begin{tabular}{|c|c|c|c|c|}
\hline Lipid Parameters & No of cases (presteroid) & Percentage $(\mathbf{n}=\mathbf{3 0})$ & No of cases & Percentage(n=30) \\
\hline Total cholesterol & 25 & 83.33 & 10 & 33.33 \\
\hline Triglyceride & 23 & 76.66 & 08 & 26.66 \\
\hline HDL & 06 & 20 & 01 & 3.33 \\
\hline LDL & 24 & 80 & 13 & 43.33 \\
\hline
\end{tabular}

The above table compares number of subjects with derangements in lipid parameters pre steroid and post steroid. After 4 weeks of steroid therapy though there were significant reductions in lipid components in all the subjects, total cholesterol was still above normal limit in $10(33.33 \%)$ subjects, triglycerides in $8(26.66 \%)$ subjects, HDL cholesterol was decreased in $1(3.33 \%)$ subject and LDL cholesterol was increased in $13(43.33 \%)$ subjects.

Table-5: Showing number of subjects with derangement of lipid parameters in subjects in remission.

\begin{tabular}{|c|c|c|}
\hline Lipid Parameters & No of cases & Percentage(n=20) \\
\hline Total cholesterol & 00 & 00 \\
\hline Triglyceride & 03 & 15 \\
\hline HDL & 02 & 10 \\
\hline LDL & 01 & 05 \\
\hline
\end{tabular}


Editorial

Out of 20 subjects in remission, triglyceride was above normal limit in 3 (15\%) subjects, HDL cholesterol was decreased in $2(10 \%)$ subject and LDL cholesterol was increased in $1(5 \%)$ subjects. Total cholesterol was normal in all the subjects.

Table-6: Comparison of lipid profile with patients in remission with patients in relapse.

\begin{tabular}{|c|c|c|c|c|c|c|}
\hline \multirow{2}{*}{ Lipid profile } & \multicolumn{9}{|c|}{ Remission } & \multirow{2}{*}{ t Value } & \multirow{2}{*}{ P Value } \\
\cline { 2 - 6 } & \multicolumn{2}{|c|}{ Yes (n=20) } & \multicolumn{2}{c|}{ No (n=30) } & \multirow{2}{*}{} \\
\cline { 2 - 6 } & Mean & SD & Mean & SD & & \\
\hline Sr. Cholesterol & 132.90 & 30.682 & 386.87 & 143.565 & 7.77 & $<0.0001$ \\
\hline Sr. Triglyceride & 94.55 & 54.033 & 347.03 & 300.077 & 3.71 & $<0.001$ \\
\hline Sr. HDL & 41.10 & 8.032 & 54.03 & 17.641 & 3.07 & $<0.005$ \\
\hline Sr. LDL & 87.50 & 17.163 & 248.03 & 150.670 & 4.73 & $<0.0001$ \\
\hline
\end{tabular}

Serum lipid of patients in remission and relapse were compared. Total cholesterol, triglyceride, HDL cholesterol and LDL cholesterol were high in relapse and were statistically significant.

Table-7: Comparison of protein, albumin, and globulin at relapse and follow up in study group.

\begin{tabular}{|c|c|c|c|c|c|c|}
\hline \multirow{2}{*}{ Parameter } & \multicolumn{2}{|c|}{ At Relapse (n=30) } & \multicolumn{2}{|c|}{ At follow up (n=30) } & \multirow{2}{*}{ t Value } & \multirow{2}{*}{ P Value } \\
\cline { 2 - 5 } & Mean & SD & Mean & SD & & \\
\hline Sr. Protein & 5.07 & 0.79 & 6.54 & 0.51 & 13.43 & $<0.0001$ \\
\hline Sr. Albumin & 2.57 & 0.74 & 3.92 & 0.26 & 10.87 & $<0.0001$ \\
\hline Sr. Globulin & 2.53 & 0.59 & 2.72 & 0.39 & 1.54 & $>0.05$ \\
\hline
\end{tabular}

Serum proteins were evaluated after 4 weeks of steroid therapy and were found to be significantly high $(6.54 \mathrm{mg} \%)$ which was statistically significant $(\mathrm{p}<0.0001)$, serum albumin was also high $(3.92 \mathrm{mg} \%)$ and statistically significant $(\mathrm{p}<0.0001)$, Serum globulin was also high (2.72) but was statistically not significant $(\mathrm{p}>0.05)$.

Table-8: Comparison of lipid profile at relapse and after1month follow up in study group.

\begin{tabular}{|c|c|c|c|c|c|c|}
\hline \multirow{2}{*}{ Lipid profile } & \multicolumn{2}{|c|}{ At Relapse (n=30) } & \multicolumn{2}{c|}{ At follow up (n=30) } & \multirow{2}{*}{ t Value } & \multirow{2}{*}{ P Value } \\
\cline { 2 - 5 } & Mean & SD & Mean & SD & & \\
\hline Sr. Cholesterol & 386.87 & 143.565 & 194.07 & 50.316 & 8.73 & $<0.0001$ \\
\hline Sr. Triglyceride & 347.03 & 300.077 & 169.60 & 92.289 & 4.11 & $<0.0001$ \\
\hline Sr. HDL & 54.03 & 17.641 & 50.73 & 9.340 & 1.15 & $>0.05$ \\
\hline Sr. LDL & 248.03 & 150.670 & 133.73 & 57.197 & 4.95 & $<0.0001$ \\
\hline
\end{tabular}

Serum lipids were evaluated after 4 weeks of steroid therapy and were found to be significantly lower and were statistically significant.

\section{Discussion}

This study was designed to study the derangement of serum lipid profile in patients with nephrotic syndrome and to know whether any correlation exists between serum lipid and albumin as hyperlipidemia may persist in some cases, leading to increased risk of atherosclerosis in later life.

It was observed that there is inverse correlation of serum albumin with total cholesterol, LDL cholesterol and triglycerides. When serum albumin was low (mean $=2.57 \mathrm{mg} \%)$ total cholesterol $($ mean $=386.87 \mathrm{mg} \%)$ was high as observed in patients in relapse phase of nephrotic syndrome, which was statistically significant $(\mathrm{p}<0.0001)$. Inverse correlation was also found with LDL cholesterol (mean $=248.03 \mathrm{mg} \%$ ), and triglycerides $($ mean $=347.03 \mathrm{mg} \%)($ Table 1$)$.

In a study on lipoprotein metabolism in nephrotic syndrome in childhood by Oetliker et al significant negative correlations were shown between plasma albumin and cholesterol $(r=-0.85, p$ less than 0.005$)$ and between plasma albumin and low density lipoprotein $(\mathrm{LDL})$ - apoprotein $\mathrm{B}(\mathrm{ApoB})(\mathrm{r}=-0.84, \mathrm{p}$ less than 0.005)[5]. 
Editorial

We compared creatinine clearance with total cholesterol $(p>0.05)$, LDL cholesterol $(p>0.05)$, Triglyceride $(p>0.05)$ and HDL $(p>0.05)$. The correlation is statistically not significant $(\mathrm{p}>0.05)$. In a study on serum creatinine is a poor marker of GFR in nephrotic syndrome by Amanda et al they found that serum creatinine is a poor marker of GFR in nephrotic syndrome [6].

In our study there was significant rise in total cholesterol $($ mean $=386.87 \mathrm{mg} \%$ ) in patients in relapse phase of nephrotic syndrome as compared to total cholesterol (mean $=132.90 \mathrm{mg} \%$ ) in patients in remission and was statistically very significant $(\mathrm{p}<0.0001)$.

Similarly LDL cholesterol (mean $=150.67 \mathrm{mg} \%$ ), triglycerides $($ mean $=347.03 \mathrm{mg} \%$ ) in patients in relapse phase was high as compared to LDL cholesterol $($ mean $=87.50 \mathrm{mg} \%)$, triglycerides $($ mean $=94.55$ $\mathrm{mg} \%$ ) in patients in remission (table 4). This was statistically significant $(p<0.0001)$ and $(p<0.001)$ respectively. Arije et al in his study on plasma lipids and lipoproteins cholesterol distributions in nephrotic syndrome patients during short term steroid treatment, had similar observations in his study [7]. We observed a positive correlation between serum total cholesterol, LDL cholesterol and triglycerides and was statistically highly significant $(\mathrm{p}<0.001)$. It was also observed in studies on lipid abnormalities in the nephrotic syndrome by David et al [8] and low density lipoprotein levels in children with nephrotic syndrome by Benakappaet al[9].

There was direct correlation of serum albumin with HDL cholesterol observed in our study. When serum albumin was low HDL cholesterol was also low. The correlation is statistically significant $(p<0.05)$. In a study on serum lipid profiles during onset and remission of steroid sensitive nephrotic syndrome in children done by Sreenivasa B et al, they also observed inverse relation between serum albumin and cholesterol and was highly significant $(p=0.000)$.[10] Patients in relapse phase of the disease were followed up after 4 weeks of steroid therapy. There was significant reduction in total cholesterol, LDL cholesterol and triglycerides.

Total cholesterol $($ mean $=194.07 \mathrm{mg} \%$ ), LDL cholesterol $($ mean $=133.73 \mathrm{mg} \%)$, triglycerides $($ mean $=169.60 \mathrm{mg} \%$ ) were significantly lower in follow up after 4 weeks of steroid therapy (table 12) and was statistically significant with all the above parameters had $(\mathrm{p}<0.0001)$. Similar observations were also made by other researchers (Mac Lean and Robson et al [11] in their study on a simple method for determining selectivity of proteinuria, Wanner et al [12] in a study on elevated plasma lipoprotein (a) in patients with nephrotic syndrome and Joven et al [13] in a study on Pattern of hyperlipoproteinaemia in human nephrotic syndrome). After 4 weeks of steroid therapy though there were significant reductions in lipid components in all the subjects, total cholesterol was still above normal limit in $10(33.33 \%)$ subjects, triglycerides in 8 (26.66\%) subjects, HDL cholesterol was decreased in 1 $(3.33 \%)$ subject and LDL cholesterol was increased in $13(43.33 \%)$ subjects.

Of 20 subjects in remission, triglyceride was above normal limit in $3(15 \%)$ subjects, HDL cholesterol was decreased in $2(10 \%)$ subject and LDL cholesterol was increased in $1(5 \%)$ subjects. Total cholesterol was normal in all the subjects.

23 patients were in relapse and of them $18(78.25 \%)$ were frequent relapse and $1(4.35 \%)$ subject was steroid resistant nephrotic syndrome. Of all the subjects, 4 subjects in the relapse phase were found to have hypertension.

Therefore, the raised levels of various lipid parameters, frequent relapsers, steroid resistant nephrotic syndrome and children with hypertension concomitantly predispose these children for increased risk of atherosclerosis and thrombosis in future.

\section{Conclusion}

Our study shows that in nephrotic syndrome, there is generalised hyperlipidemia and hypoalbuminemia. Serum cholesterol, triglycerides and LDL cholesterol were deranged in almost all subjects in relapse phase of nephrotic syndrome. Few subjects in remission phase also had deranged cholesterol, triglycerides and LDL cholesterol.

In cases of relapse after 4 weeks of steroid therapy there is persistent raised total cholesterol (33.33\%), triglycerides (26.66\%) and LDL (43.3\%) cholesterol, which may predispose to the development of atherosclerosis in near future. However, all the children require long term follow-up before any conclusion can be drawn.

What does this study add? The raised levels of various lipid parameters, frequent relapsers, steroid resistant nephrotic syndrome and children with hypertension concomitantly predispose these children for increased risk of atherosclerosis and thrombosis in future. 
Editorial

Atherosclerosis occurs due to narrowing of vessels which takes years to develop. However, the process of atherosclerosis begins in childhood, is usually mild and progresses over a period of time. So it is unusual for children or teenagers to have a heart attack or stroke as a result of atherosclerosis. In some children, atherosclerosis worsens rapidly, increasing the risk of heart disease, and less commonly, stroke in early adult life. Long term follow up of these patients are required, for detection of complications if any, and possibility of therapeutic interventions.

Contribution by authors: Dr. Anshuman Singh and Dr.SuhasSodal have collected the data. Dr Renuka Jadhav have compiled the data. Dr. Sanjay Chavan and Dr. Shradha Salunkhe have analyzed the data and written the manuscript. Dr Sharad Agarkhedkar(H.O.D) has constantly guided throughout the study.

Acknowledgements: I am thankful to the children involved in this study and their parents, without their cooperation it would not have been possible to complete this study.

\section{Declarations}

Funding: Nil, Conflict of interest: None initiated, Perission from IRB: Yes

Ethical Approval: The study was carried out after the approval from the Institutional Ethical Committee of Dr. D.Y. Patil Medical College, Hospital and Research Center, Pimpri, Pune.

\section{References}

1. Epstein AA. The nature and treatment of Nephrosis. JAMA 1917; 69: 444-47

2. Bhandari B, Mandowara SL. Lipoprotein profile in nephrotic syndrome. Indian Pediatr. 1980 May;17(5): 416-9.

3. El-Melegy NT, Mohamed NA, Sayed MM. Oxidative modification of low density lipoprotein in relation to dyslipidemia and oxidant status in children with steroid sensitive nephrotic syndrome. Pediatr Res. 2008 Apr;63 (4): 404-9
4. Ksiazek J, Ciechanowicz A, Wierzbicka A, et. el. Is dyslipidemia sustained during remission of nephritic syndrome genetically determined? Evaluation of genetic polymorphisms of proteins involved in lipoprotein metabolism in childrenand adolescents with nephrotic syndrome. Pol Arch Med Wewn. 2009 Jan-Feb; 119 (1-2): 11-6.

5. Oetliker OH, Mordasini R, Lütschg J, et al. Lipoprotein metabolism in nephrotic syndrome in childhood. Pediatr Res. 1980 Jan;14(1):64-6.

6. Branten AJ, Vervoort G, Wetzels JF. Serum creatinine is a poor marker of GFR in nephrotic syndrome. Nephrol Dial Transplant. 2005 Apr; 20 (4): 707- 11. Epub 2005 Feb 15.

7. Arije A, Erasmus RT, Anjorin SA: Plasma lipids and lipoproteins cholesterol distributions in nephrotic syndrome patients during short term steroid treatment. Cent Afr J Med 1993; 39 (10): 211-5

8. Wheeler DC, Bernard DB. Lipid abnormalities in the nephrotic syndrome: causes, consequences, and treatment. Am J Kidney Dis. 1994 Mar;23(3):331-46.

9. Benakappa DG, Subba Rao A, Sastry NSC: Low density lipoprotein levels in children with nephrotic syndrome; Indian paediatrics 1976; 13(4); 287-89.

10. Sreenivasa B, Sunil Kumar, Suresh Babu MT, Ragavendra K. Serum lipid profiles during onset and remission of steroid sensitive nephrotic syndrome in children; MD 49; Volume 1; Issue 1; 2015; 1-4

11. MacLean PR, Robson JS. A simple method for determining selectivity of proteinuria. Lancet. 1967 Mar $11 ; 1(7489): 539-42$.

12. Wanner C, Rader D, Bartens W, et al. Wieland H. Elevated plasma lipoprotein (a) in patients with the nephrotic syndrome. Ann Intern Med. 1993 Aug 15;119 (4): 263-9.

13. Joven J, Villabona C, Vilella E. Pattern of hyper lipoproteinaemia in human nephrotic syndrome: Influence of renal failure and diabetes mellitus. Nephron 1993; 64(4); 565-69.

\section{How to cite this article?}

Chavan S, Salunkhe S, Singh A, Agarkhedkar S, Suhas Sodal, Jadhav R. Lipid profile in children with Nephrotic syndrome. Int J Pediatr Res. 2018;5(6):314-319.doi:10.17511/ijpr.2018.i06.03. 